\title{
A Topological Coincidence Theory for Multifunctions via Homotopy
}

\section{Donal O'Regan}

School of Mathematics, Statistics and Applied Mathematics, National University of Ireland, Galway H91 TK33, Ireland; donal.oregan@nuigalway.ie

Received: 21 February 2020; Accepted: 12 March 2020; Published: 16 March 2020

Abstract: A new simple result is presented which immediately yields the topological transversality theorem for coincidences.

Keywords: essential maps; homotopy

\section{Introduction}

The topological transversality theorem of Granas [1] states that if $F$ and $G$ are continuous compact single valued maps and $F \cong G$ then $F$ is essential if and only if $G$ is essential. These concepts were generalized to multimaps (compact and noncompact) and for $\Phi$-essential maps in a general setting (see $[2,3]$ and the references therein). In this paper we approach this differently and we present a very general topological transversality theorem for coincidences.

For convenience we desribe now a class of maps one could consider in this setting. Let $X$ and $Z$ be subsets of Hausdorff topological spaces. We will consider maps $F: X \rightarrow K(Z)$; here $K(Z)$ denotes the family of nonempty compact subsets of $Z$. A nonempty topological space is said to be acyclic if all its reduced Čech homology groups over the rationals are trivial. Now $F: X \rightarrow K(Z)$ is called acyclic if $F$ has acyclic values.

\section{Topological Transversality Theorem}

In this paper we will consider two classes $\mathbf{A}$ and $\mathbf{B}$ of maps. These are abstract classes which include many types of maps in the literature (see Remark 1). Let $E$ be a completely regular space (i.e., a Tychonoff space) and $U$ an open subset of $E$. We let $\bar{U}$ (respectively, $\partial U$ ) denote the closure (respectively, the boundary) of $U$ in $E$.

Definition 1. We say $F \in A(\bar{U}, E)$ if $F \in \mathbf{A}(\bar{U}, E)$ and $F: \bar{U} \rightarrow K(E)$ is a upper semicontinuous (u.s.c.) compact map.

Remark 1. Examples of $F \in \mathbf{A}(\bar{U}, E)$ might be that $F: \bar{U} \rightarrow K(E)$ has convex values or $F: \bar{U} \rightarrow K(E)$ has acyclic values.

In this paper we fix a $\Phi \in B(\bar{U}, E)$ (i.e., $\Phi \in \mathbf{B}(\bar{U}, E)$ and $\Phi: \bar{U} \rightarrow K(E)$ is a u.s.c. map).

Definition 2. We say $F \in A_{\partial U}(\bar{U}, E)$ if $F \in A(\bar{U}, E)$ and $F(x) \cap \Phi(x)=\varnothing$ for $x \in \partial U$.

Next we consider homotopy for maps in $A_{\partial U}(\bar{U}, E)$. We present two interpretations.

Definition 3. Two maps $F, G \in A_{\partial U}(\bar{U}, E)$ are said to be homotopic in $A_{\partial U}(\bar{U}, E)$, written $F \cong G$ in $A_{\partial U}(\bar{U}, E)$, if there exists a u.s.c. compact map $\Psi: \bar{U} \times[0,1] \rightarrow K(E)$ with $\Psi(\cdot, \eta(\cdot)) \in \mathbf{A}(\bar{U}, E)$ for any 
continuous function $\eta: \bar{U} \rightarrow[0,1]$ with $\eta(\partial U)=0, \Phi(x) \cap \Psi_{t}(x)=\varnothing$ for any $x \in \partial U$ and $t \in(0,1)$ (here $\left.\Psi_{t}(x)=\Psi(x, t)\right), \Psi_{0}=F$ and $\Psi_{1}=G$.

Remark 2. Alternatively we could use the following definition for $\cong$ in $A_{\partial u}(\bar{U}, E): F \cong G$ in $A_{\partial u}(\bar{U}, E)$ if there exists a u.s.c. compact map $\Psi: \bar{U} \times[0,1] \rightarrow K(E)$ with $\Psi \in \mathbf{A}(\bar{U} \times[0,1], E), \Phi(x) \cap \Psi_{t}(x)=\varnothing$ for any $x \in \partial U$ and $t \in(0,1)$ (here $\left.\Psi_{t}(x)=\Psi(x, t)\right), \Psi_{0}=F$ and $\Psi_{1}=G$. If we use this definition then we always assume for any map $\Theta \in \mathbf{A}(\bar{U} \times[0,1], E)$ and any map $f \in \mathbf{C}(\bar{U}, \bar{U} \times[0,1])$ then $\Theta \circ f \in \mathbf{A}(\bar{U}, E)$; here $\mathbf{C}$ denotes the class of single valued continuous functions.

Definition 4. Let $F \in A_{\partial U}(\bar{U}, E)$. We say $F$ is $\Phi$-essential in $A_{\partial U}(\bar{U}, E)$ if for every map $J \in A_{\partial U}(\bar{U}, E)$ with $\left.J\right|_{\partial U}=\left.F\right|_{\partial U}$ there exists a $x \in U$ with $\Phi(x) \cap J(x) \neq \varnothing$.

We now present a simple result. From this result the topological transversality theorem will be immediate. In our next theorem $E$ will be a completely regular topological space and $U$ will be an open subset of $E$.

Theorem 1. Let $F \in A_{\partial U}(\bar{U}, E)$ and let $G \in A_{\partial U}(\bar{U}, E)$ be $\Phi$-essential in $A_{\partial U}(\bar{U}, E)$. Also suppose

$$
\left\{\begin{array}{l}
\text { for any map } J \in A_{\partial U}(\bar{U}, E) \text { with }\left.J\right|_{\partial U}=\left.F\right|_{\partial U} \\
\text { we have } G \cong J \text { in } A_{\partial U}(\bar{U}, E) .
\end{array}\right.
$$

Then $F$ is $\Phi-$ essential in $A_{\partial U}(\bar{U}, E)$.

Proof. In the proof below we assume $\cong$ in $A_{\partial U}(\bar{U}, E)$ is as in Definition 3. Let $J \in A_{\partial U}(\bar{U}, E)$ with $\left.J\right|_{\partial U}=\left.F\right|_{\partial U}$. From (1) there exists a u.s.c. compact map $H^{J}: \bar{U} \times[0,1] \rightarrow K(E)$ with $H^{J}(\cdot, \eta(\cdot)) \in$ $\mathbf{A}(\bar{U}, E)$ for any continuous function $\eta: \bar{U} \rightarrow[0,1]$ with $\eta(\partial U)=0, \Phi(x) \cap H_{t}^{J}(x)=\varnothing$ for any $x \in \partial U$ and $t \in(0,1)$ (here $\left.H_{t}^{J}(x)=H^{J}(x, t)\right), H_{0}^{J}=G$ and $H_{1}^{J}=J$. Let

$$
K=\left\{x \in \bar{U}: \Phi(x) \cap H^{J}(x, t) \neq \varnothing \text { for some } t \in[0,1]\right\}
$$

and

$$
D=\left\{(x, t) \in \bar{U} \times[0,1]: \Phi(x) \cap H^{J}(x, t) \neq \varnothing\right\} .
$$

Now $D \neq \varnothing$ (note $G$ is $\Phi$-essential in $\left.A_{\partial U}(\bar{U}, E)\right)$ and $D$ is closed (note $\Phi$ and $H^{J}$ are u.s.c.) and so $D$ is compact (note $H^{J}$ is a compact map). Let $\pi: \bar{U} \times[0,1] \rightarrow \bar{U}$ be the projection. Now $K=\pi(D)$ is closed (see Kuratowski's theorem ([4], p. 126) and so in fact compact (recall projections are continuous). Also note $K \cap \partial U=\varnothing$ (since $\Phi(x) \cap H_{t}^{J}(x)=\varnothing$ for any $x \in \partial U$ and $\left.t \in[0,1]\right)$ so since $E$ is Tychonoff there exists a continuous map (called the Urysohn map) $\mu: \bar{U} \rightarrow[0,1]$ with $\mu(\partial U)=0$ and $\mu(K)=1$. Let $R(x)=H^{J}(x, \mu(x))$. Now $R \in A_{\partial U}(\bar{U}, E)$ with $\left.R\right|_{\partial U}=\left.G\right|_{\partial U}$ (note if $x \in \partial U$ then $R(x)=H^{J}(x, 0)=G(x)$ and $\left.R(x) \cap \Phi(x)=G(x) \cap \Phi(x)\right)$. Now since $G$ is $\Phi$-essential in $A_{\partial U}(\bar{U}, E)$ there exists a $x \in U$ with $\Phi(x) \cap R(x) \neq \varnothing$ (i.e., $\left.\Phi(x) \cap H_{\mu(x)}^{J}(x) \neq \varnothing\right)$. Thus $x \in K$ so $\mu(x)=1$ and $\Phi(x) \cap H_{1}^{J}(x) \neq \varnothing$ that is, $\Phi(x) \cap J(x) \neq \varnothing$.

Remark 3. (i). In the proof of Theorem 1 it is simple to adjust the proof if we use $\cong$ in $A_{\partial U}(\bar{U}, E)$ from Remark 2 if we note $H^{J}(x, \mu(x))=H^{J} \circ g(x)$ where $g: \bar{U} \rightarrow \bar{U} \times[0,1]$ is given by $g(x)=(x, \mu(x))$.

(ii). One could replace u.s.c. in the Definition of $A(\bar{U}, E), B(\bar{U}, E)$, Definition 3 and Remark 2 with any condition that guarantees that $K$ in the proof of Theorem 1 is closed; this is all that is needed if $E$ is normal. If $E$ is Tychonoff and not normal the one can also replace the compactness of the map in $A(\bar{U}, E)$, Definition 3 and Remark 2 with any condition that guarantees that $K$ in the proof of Theorem 1 is compact.

(iii). Theorem 1 immediately yields a general Leray-Schauder type alternative for coincidences. Let $E$ be a completely metrizable locally convex space, $U$ an open subset of $E, F \in A_{\partial U}(\bar{U}, E), G \in A_{\partial U}(\bar{U}, E)$ is 
$\Phi$-essential in $A_{\partial U}(\bar{U}, E), \Phi(x) \cap[t F(x)+(1-t) G(x)]=\varnothing$ for $x \in \partial U$ and $t \in(0,1)$, and $\eta(\cdot) J(\cdot)+$ $(1-\eta(\cdot)) G(\cdot) \in \mathbf{A}(\bar{U}, E)$ for any continuous function $\eta: \bar{U} \rightarrow[0,1]$ with $\eta(\partial U)=0$ and any map $J \in A_{\partial U}(\bar{U}, E)$ with $\left.J\right|_{\partial U}=\left.F\right|_{\partial U}$. Then $F$ is $\Phi$-essential in $A_{\partial U}(\bar{U}, E)$.

The proof is immediate from Theorem 1 since topological vector spaces are completely regular and note if $J \in A_{\partial U}(\bar{U}, E)$ with $\left.J\right|_{\partial U}=\left.F\right|_{\partial U}$ then with $H^{J}(x, t)=t J(x)+(1-t) G(x)$ note $H_{0}^{J}=G, H_{1}^{J}=J$, $H^{J}: \bar{U} \times[0,1] \rightarrow K(E)$ is a u.s.c. compact (see [5], Theorem 4.18) map, and $H^{J}(\cdot, \eta(\cdot)) \in \mathbf{A}(\bar{U}, E)$ for any continuous function $\eta: \bar{U} \rightarrow[0,1]$ and $\Phi(x) \cap H_{t}^{J}(x)=\varnothing$ for $x \in \partial U$ and $t \in(0,1)$ (if $x \in \partial U$ and $t \in(0,1)$ then since $\left.J\right|_{\partial U}=\left.F\right|_{\partial U}$ we note that $\left.\Phi(x) \cap H_{t}^{J}(x)=\Phi(x) \cap[t F(x)+(1-t) G(x)]\right)$ so as a result $G \cong J$ in $A_{\partial U}(\bar{U}, E)$ (i.e., (1) holds). (Note E being a completely metrizable locally convex space can be replaced by any (Hausdorff) topological vector space $E$ if the space $E$ has the property that the closed convex hull of a compact set in $E$ is compact. In fact it is easy to see, if we argue differently, that all we need to assume is that $E$ is a topological vector space).

With this simple result we now present the topological transversality theorem. Assume

$$
\cong \text { in } A_{\partial U}(\bar{U}, E) \text { is an equivalence relation }
$$

and

$$
\text { if } F, G \in A_{\partial U}(\bar{U}, E) \text { with }\left.F\right|_{\partial U}=\left.G\right|_{\partial U} \text { then } F \cong G \text { in } A_{\partial U}(\bar{U}, E) \text {. }
$$

In our next theorem $E$ will be a completely regular topological space and $U$ will be an open subset of $E$.

Theorem 2. Assume (2) and (3) hold. Suppose $F$ and $G$ are two maps in $A_{\partial U}(\bar{U}, E)$ with $F \cong G$ in $A_{\partial U}(\bar{U}, E)$. Now $F$ is $\Phi$-essential in $A_{\partial U}(\bar{U}, E)$ if and only if $G$ is $\Phi$-essential in $A_{\partial U}(\bar{U}, E)$.

Proof. Assume $G$ is $\Phi$-essential in $A_{\partial U}(\bar{U}, E)$. Let $J \in A_{\partial U}(\bar{U}, E)$ with $\left.J\right|_{\partial U}=\left.F\right|_{\partial U}$. We will show $G \cong J$ in $A_{\partial U}(\bar{U}, E)$ (i.e., we will show (1)) and then Theorem 1 guarantees that $F$ is $\Phi$-essential in $A_{\partial U}(\bar{U}, E)$. Note $G \cong J$ in $A_{\partial U}(\bar{U}, E)$ is immediate since from (3) we have $J \cong F$ in $A_{\partial U}(\bar{U}, E)$ and since $F \cong G$ in $A_{\partial U}(\bar{U}, E)$ then (2) guarantees that $G \cong J$ in $A_{\partial U}(\bar{U}, E)$. Similarly if $F$ is $\Phi$-essential in $A_{\partial U}(\bar{U}, E)$ then $G$ is $\Phi$-essential in $A_{\partial U}(\bar{U}, E)$.

Remark 4. Suppose $E$ is a (Hausdorff) topological vector space, $U$ is a open convex subset of $E$ and $F \in \mathbf{A}(\bar{U}, E)$ means $F: \bar{U} \rightarrow K(E)$ has acyclic values then immediately (2) holds (we use the definition of $\cong$ in $A_{\partial U}(\bar{U}, E)$ from Definition 3). Suppose

$$
\text { there exists a retraction } r: \bar{U} \rightarrow \partial U \text {. }
$$

(Note (4) is satisfied if $E$ is an infinite dimensional Banach space).

Then (3) holds (we use the definition of $\cong$ in $A_{\partial U}(\bar{U}, E)$ from Definition 3). To see this let $r$ be in (4), $F, G \in A_{\partial U}(\bar{U}, E)$ with $\left.F\right|_{\partial U}=\left.G\right|_{\partial U}$. Consider $F^{\star}$ given by $F^{\star}(x)=F(r(x)), x \in \bar{U}$. Note $F^{\star}(x)=$ $G(r(x)), x \in \bar{U}$ since $\left.F\right|_{\partial u}=\left.G\right|_{\partial u}$. Now take

$$
\Lambda(x, \lambda)=G(2 \lambda r(x)+(1-2 \lambda) x)=G \circ j(x, \lambda) \text { for }(x, \lambda) \in \bar{U} \times\left[0, \frac{1}{2}\right]
$$

(here $j: \bar{U} \times\left[0, \frac{1}{2}\right] \rightarrow \bar{U}$ (note $\bar{U}$ is convex) is given by $j(x, \lambda)=2 \lambda r(x)+(1-2 \lambda) x$ ) it is easy to see that

$$
G \cong F^{\star} \text { in } A_{\partial U}(\bar{U}, E)
$$

note $\Lambda: \bar{U} \times\left[0, \frac{1}{2}\right] \rightarrow K(E)$ is a u.s.c. compact map and also for a fixed $x \in \bar{U}$ note $\Lambda(x, \mu(x))=$ $G(j(x, \mu(x)))$ has acyclic values and so $\Lambda(\cdot, \eta(\cdot)) \in \mathbf{A}(\bar{U}, E)$ for any continuous function $\eta: \bar{U} \rightarrow[0,1]$ 
with $\eta(\partial U)=0$, and finally note $\Phi(x) \cap \Lambda_{t}(x)=\varnothing$ for $x \in \partial U$ and $t \in\left[0, \frac{1}{2}\right]$ (note if $x \in \partial U$ and $t \in\left[0, \frac{1}{2}\right]$ then since $r(x)=x$ we have $\Phi(x) \cap \Lambda_{t}(x)=\Phi(x) \cap G(x)$ ). Similarly with

$$
\Theta(x, \lambda)=F((2-2 \lambda) r(x)+(2 \lambda-1) x) \text { for }(x, \lambda) \in \bar{U} \times\left[\frac{1}{2}, 1\right]
$$

it is easy to see that

$$
F^{\star} \cong F \text { in } A_{\partial U}(\bar{U}, E) .
$$

Consequently $F \cong G$ in $A_{\partial U}(\bar{U}, E)$ so (3) holds.

It is easy to present examples of $\Phi$-essential maps if one uses coincidence result from the literature. In our next theorem $E$ will be a (Hausdorff) topological space and $U$ will be an open subset of $E$.

Theorem 3. Let $\Phi \in B(\bar{U}, E)$ and $F \in A_{\partial U}(\bar{U}, E)$. Assume the following conditions hold:

$$
\left\{\begin{array}{l}
\text { there exists a retraction } r: E \rightarrow \bar{U} \\
\text { with } r(w) \in \partial U \text { if } w \in E \backslash U
\end{array}\right.
$$

and

$$
\left\{\begin{array}{l}
\text { for any map } J \in A_{\partial U}(\bar{U}, E) \text { with }\left.J\right|_{\partial U}=\left.F\right|_{\partial U} \\
\text { (i). there exists } a \quad w \in \bar{U} \text { with } r J(w) \cap \Phi(w) \neq \varnothing \text {, and } \\
\text { (ii). there is no } z \in E \backslash U \text { and } y \in \bar{U} \text { with } z \in J(y) \text { and } r(z) \in \Phi(y) .
\end{array}\right.
$$

Then $F$ is $\Phi$-essential in $A_{\partial U}(\bar{U}, E)$.

Proof. Let $J \in A_{\partial U}(\bar{U}, E)$ with $\left.J\right|_{\partial U}=\left.F\right|_{\partial U}$. Now (6) (i) implies there exists a $w \in \bar{U}$ with $r J(w) \cap$ $\Phi(w) \neq \varnothing$. Then there exists a $z \in J(w)$ with $r(z) \in \Phi(w)$. Note $z \in E \backslash U$ or $z \in U$. If $z \in E \backslash U$ then $z \in J(w), w \in \bar{U}$ and $r(z) \in \Phi(w)$ which contradicts (6) (ii). Thus $z \in U$ so $r(z)=z$ and as a result $z \in J(w)$ and $z(=r(z)) \in \Phi(w)$ that is, $\Phi(w) \cap J(w) \neq \varnothing$.

Remark 5. (i). Suppose $\Phi=i$ (identity) and $F \in \mathbf{A}(\bar{U}, E)$ means $F: \bar{U} \rightarrow K(E)$ has acyclic values. Then (6) (i) holds (i.e., there exists $a w \in \bar{U}$ with $w \in r J(w)$ ) from a theorem of Eilenberg and Montgomery [6,7] (note $r$ is continuous and $J$ is an acyclic u.s.c. compact map).

(ii). Now let us consider (5) and (6) (ii). Now in addition assume $E$ is a locally convex topological vector space, $0 \in U$ and $U$ an open convex subset of $E$. Let

$$
r(x)=\frac{x}{\max \{1, \mu(x)\}} \text { for } x \in E,
$$

where $\mu$ is the Minkowski functional on $\bar{U}$ (i.e., $\mu(x)=\inf \{\alpha>0: x \in \alpha \bar{U}\}$ ). Now (5) holds

First let $\Phi=i$. If we assume a Leray-Schauder type condition

$$
x \notin \lambda F(x) \text { for } x \in \partial U \text { and } \lambda \in(0,1)
$$

then (6) (ii) holds. To see this let $J \in A_{\partial U}(\bar{U}, E)$ with $\left.J\right|_{\partial U}=\left.F\right|_{\partial U}$. Suppose there is $a z \in E \backslash U$ and $y \in \bar{U}$ with $z \in J(y)$ and $r(z) \in \Phi(y)$ (i.e $r(z)=y$ since $\Phi=i)$. Now

$$
y=r(z)=\frac{z}{\mu(z)} \text { with } \mu(z) \geq 1 \text { since } z \in E \backslash U,
$$

so $y \in \lambda J(y)$ with $0<\lambda=\frac{1}{\mu(y)} \leq 1$. Note $y=r(z) \in \partial U$ since $z \in E \backslash U$ so $y \in \lambda F(y)$ since $\left.J\right|_{\partial U}=\left.F\right|_{\partial U}$. This contradicts (7). 
Next we do not assume $\Phi=i$. Assume

$$
\left\{\begin{array}{l}
\text { for any map } J \in A_{\partial U}(\bar{U}, E) \text { with }\left.J\right|_{\partial U}=\left.F\right|_{\partial U} \\
\text { if } y \in \bar{U}, z \in E \backslash U \text { with } z \in J(y) \\
\text { and } r(z) \in \Phi(y) \text { then } y \in \partial U
\end{array}\right.
$$

and

$$
\Phi(x) \cap \lambda F(x)=\varnothing \text { for } x \in \partial U \text { and } \lambda \in(0,1) \text {. }
$$

Then (6) (ii) holds. To see this let $J \in A_{\partial U}(\bar{U}, E)$ with $\left.J\right|_{\partial U}=\left.F\right|_{\partial U}$. Suppose there is $a z \in E \backslash U$ and $y \in \bar{U}$ with $z \in J(y)$ and $r(z) \in \Phi(y)$. Now (8) guarantees that $y \in \partial U$. Also $r(z)=\frac{z}{\mu(z)}$ with $\mu(z) \geq 1$, so $r(z) \in \Phi(y)$ and $r(z) \in \frac{1}{\mu(z)} J(y)$. Thus $\varnothing \neq \Phi(y) \cap \lambda J(y)=\Phi(y) \cap \lambda F(y)$ (since J| $\left.\left.\right|_{\partial U}=\left.F\right|_{\partial U}\right)$ with $0<\lambda=\frac{1}{\mu(y)} \leq 1$, and this contradicts (9).

(iii). One also has a "dual" version of Theorem 3 if we consider J $r$ instead of $r$ J. Let $\Phi \in B(E, E)$ (i.e., $\Phi \in \mathbf{B}(E, E)$ and $\Phi: E \rightarrow K(E)$ is a u.s.c. map), $F \in A_{\partial U}(\bar{U}, E)$ and assume (5) holds. In addition suppose

$$
\left\{\begin{array}{l}
\text { for any map } J \in A_{\partial U}(\bar{U}, E) \text { with }\left.J\right|_{\partial U}=\left.F\right|_{\partial U} \\
\text { there exists } a w \in E \text { with } J r(w) \cap \Phi(w) \neq \varnothing
\end{array}\right.
$$

and

$$
\left\{\begin{array}{l}
\text { there is no } y \in E \backslash U \text { and } z \in \partial U \text { with } \\
z=r(y) \text { and } F(z) \cap \Phi(y) \neq \varnothing .
\end{array}\right.
$$

Then $F$ is $\Phi$-essential in $A_{\partial u}(\bar{U}, E)$.

The proof is immediate since for any $J \in A_{\partial U}(\bar{U}, E)$ with $\left.J\right|_{\partial U}=\left.F\right|_{\partial U}$ from (10) there exists a $y \in E$ with $J r(y) \cap \Phi(y) \neq \varnothing$, so if $z=r(y)$ then $J(z) \cap \Phi(y) \neq \varnothing$. If $y \in E \backslash U$ then $z \in \partial U$ and $\varnothing \neq J(z) \cap \Phi(y)=$ $F(z) \cap \Phi(y)$ (since $\left.J\right|_{\partial U}=\left.F\right|_{\partial U}$ ), a contradiction. Thus $y \in U$ so $z=r(y)=y$ and $J(y) \cap \Phi(y) \neq \varnothing$.

In our next theorem $E$ will be a (Hausdorff) topological space and $U$ will be an open subset of $E$.

Theorem 4. Let $\Phi \in B(E, E)$ and assume:

$$
0 \in \mathbf{A}(\bar{U}, E) \text { where } 0 \text { denotes the zero map }
$$

$$
\left\{\begin{array}{l}
\text { for any map } J \in A_{\partial U}(\bar{U}, E) \text { with }\left.J\right|_{\partial U}=\{0\} \text { and } \\
R(x)=\left\{\begin{array}{l}
J(x), x \in \bar{U} \\
\{0\}, x \in E \backslash \bar{U}
\end{array}\right. \\
\text { there exists a } y \in E \text { with } \Phi(y) \cap R(y) \neq \varnothing
\end{array}\right.
$$

and

$$
\text { there is no } z \in E \backslash U \text { with } \Phi(z) \cap\{0\} \neq \varnothing \text {. }
$$

Then the zero map is $\Phi$-essential in $A_{\partial U}(\bar{U}, E)$.

Proof. Note $0 \in A_{\partial U}(\bar{U}, E)$ (see (12) and (14)). Let $J \in A_{\partial U}(\bar{U}, E)$ with $\left.J\right|_{\partial U}=\{0\}$. Let $R$ be as in (13) so there exists a $y \in E$ with $\Phi(y) \cap R(y) \neq \varnothing$. We have two cases, namely $y \in U$ and $y \in E \backslash U$. If $y \in E \backslash U$ then $R(y)=\{0\}$ so $\Phi(y) \cap\{0\} \neq \varnothing$, and this contradicts (14). Thus $y \in U$ so $\Phi(y) \cap J(y) \neq \varnothing$.

Remark 6. (i). Suppose $F \in \mathbf{A}(\bar{U}, E)$ means $F: \bar{U} \rightarrow K(E)$ has acyclic values. If $\Phi \in B(E, E)$ and (13) and (14) are satisfied then Theorem 4 guarantees that zero map is $\Phi$-essential in $A_{\partial U}(\bar{U}, E)$.

Suppose $E$ is a completely metrizable locally convex space, $U$ is an open convex subset of $E, 0 \in U$, $F \in A_{\partial U}(\bar{U}, E), \Phi \in B(E, E)$ and assume (4), (9) (namely $\Phi(x) \cap \lambda F(x)=\varnothing$ for $x \in \partial U$ and $\lambda \in(0,1)$ ), 
(13) and (14) hold. Then Theorem 2 and Remark 4 guarantees that $F$ is $\Phi$-essential in $A_{\partial U}(\bar{U}, E)$. This is immediate since a homotopy (Definition 3) from $F$ to $\{0\}$ is $\Psi(x, t)=t F(x)$ (here $t \in[0,1]$ and $x \in \bar{U}$ ). To see this note $\Psi: \bar{U} \times[0,1] \rightarrow K(E)$ is a upper semicontinuous compact (see [5], Theorem 4.18) map and also note for a fixed $t \in[0,1]$ and a fixed $x \in \bar{U}$ that $\Psi_{t}(x)$ is acyclic valued (recall homeomorphic spaces have isomorphic homology groups) so $\Psi_{t} \in A_{\partial U}(\bar{U}, E)$ and this immediately implies $\Psi(\cdot, \eta(\cdot)) \in \mathbf{A}(\bar{U}, E)$ for any continuous function $\eta: \bar{U} \rightarrow[0,1], \eta(\partial U)=0$ since for $x \in \bar{U}$ fixed note $\Psi(x, \mu(x))=\Psi_{\mu(x)}(x)=\Psi_{t}(x)$ with $t=\mu(x) \in[0,1]$. Note $E$ being a completely metrizable locally convex space can be replaced by any (Hausdorff) topological vector space $E$ if the space E has the property that the closed convex hull of a compact set in $E$ is compact. In fact it is easy to see, if we argue differently, that all we need to assume is that $E$ is a topological vector space.

(ii). It is very easy to extend the above ideas to the $(L, T) \Phi$-essential maps in [2].

Now we consider $d-\Phi-$ essential maps. Let $E$ be a completely regular topological space and $U$ an open subset of $E$. For any map $F \in A(\bar{U}, E)$ write $F^{\star}=I \times F: \bar{U} \rightarrow K(\bar{U} \times E)$, with $I: \bar{U} \rightarrow \bar{U}$ given by $I(x)=x$, and let

$$
d:\left\{\left(F^{\star}\right)^{-1}(B)\right\} \cup\{\varnothing\} \rightarrow \Omega
$$

be any map with values in the nonempty set $\Omega$ where $B=\{(x, \Phi(x)): x \in \bar{U}\}$.

Definition 5. Let $F \in A_{\partial U}(\bar{U}, E)$ and write $F^{\star}=I \times F$. We say $F^{\star}: \bar{U} \rightarrow K(\bar{U} \times E)$ is d- $\Phi-$ essential if for every map $J \in A_{\partial U}(\bar{U}, E)$ (write $\left.J^{\star}=I \times J\right)$ with $\left.J\right|_{\partial U}=\left.F\right|_{\partial U}$ we have that $d\left(\left(F^{\star}\right)^{-1}(B)\right)=$ $d\left(\left(J^{\star}\right)^{-1}(B)\right) \neq d(\varnothing)$.

Remark 7. If $F^{\star}$ is $d-\Phi-e s s e n t i a l$ then

$$
\varnothing \neq\left(F^{\star}\right)^{-1}(B)=\{x \in \bar{U}:(x, F(x)) \cap(x, \Phi(x)) \neq \varnothing\},
$$

so there exists a $x \in U$ with $(x, \Phi(x)) \cap(x, F(x)) \neq \varnothing$ (i.e., $\Phi(x) \cap F(x) \neq \varnothing)$.

In our next theorem $E$ will be a completely regular topological space and $U$ will be an open subset of $E$.

Theorem 5. Let $B=\{(x, \Phi(x)): x \in \bar{U}\}$, $d$ is defined in (15), $F \in A_{\partial U}(\bar{U}, E)$ and $G \in A_{\partial U}(\bar{U}, E)$ (write $F^{\star}=I \times F$ and $\left.G^{\star}=I \times G\right)$. Suppose $G^{\star}$ is $d-\Phi-$ essential and

$$
\left\{\begin{array}{l}
\text { for any map } J \in A_{\partial U}(\bar{U}, E) \text { with }\left.J\right|_{\partial U}=\left.F\right|_{\partial U} \\
\text { we have } G \cong J \text { in } A_{\partial U}(\bar{U}, E) \text { and } \\
d\left(\left(F^{\star}\right)^{-1}(B)\right)=d\left(\left(G^{\star}\right)^{-1}(B)\right) .
\end{array}\right.
$$

Then $F^{\star}$ is $d-\Phi-e s s e n t i a l$.

Proof. In the proof below we assume $\cong$ in $A_{\partial U}(\bar{U}, E)$ is as in Definition 3. Consider any map $J \in$ $A_{\partial U}(\bar{U}, E)$ (write $\left.J^{\star}=I \times J\right)$ and $\left.J\right|_{\partial U}=\left.F\right|_{\partial U}$. From (16) there exists a u.s.c. compact map $H^{J}$ : $\bar{U} \times[0,1] \rightarrow K(E)$ with $H^{J}(\cdot, \eta(\cdot)) \in \mathbf{A}(\bar{U}, E)$ for any continuous function $\eta: \bar{U} \rightarrow[0,1]$ with $\eta(\partial U)=0, \Phi(x) \cap H_{t}^{J}(x)=\varnothing$ for any $x \in \partial U$ and $t \in(0,1)$ (here $\left.H_{t}^{J}(x)=H^{J}(x, t)\right), H_{0}^{J}=G$, $H_{1}^{J}=J$ and $d\left(\left(F^{\star}\right)^{-1}(B)\right)=d\left(\left(G^{\star}\right)^{-1}(B)\right)$. Let $\left(H^{J}\right)^{\star}: \bar{U} \times[0,1] \rightarrow K(\bar{U} \times E)$ be given by $\left(H^{J}\right)^{\star}(x, t)=\left(x, H^{J}(x, t)\right)$ and let

$$
K=\left\{x \in \bar{U}:(x, \Phi(x)) \cap\left(H^{J}\right)^{\star}(x, t) \neq \varnothing \text { for some } t \in[0,1]\right\} .
$$


Now $K \neq \varnothing$ is closed, compact and $K \cap \partial U=\varnothing$ so since $E$ is Tychonoff there exists a Urysohn map $\mu: \bar{U} \rightarrow[0,1]$ with $\mu(\partial U)=0$ and $\mu(K)=1$. Let $R(x)=H^{J}(x, \mu(x))$ and write $R^{\star}=I \times R$. Now $R \in A_{\partial U}(\bar{U}, E)$ (if $x \in \partial U$ then $\mu(x)=0$ so $R(x)=G(x)$ ) with $\left.R\right|_{\partial U}=\left.G\right|_{\partial U}$. Since $G^{\star}$ is $d-\Phi-$ essential then

$$
d\left(\left(G^{\star}\right)^{-1}(B)\right)=d\left(\left(R^{\star}\right)^{-1}(B)\right) \neq d(\varnothing)
$$

Now since $\mu(K)=1$ we have

$$
\begin{aligned}
\left(R^{\star}\right)^{-1}(B) & =\left\{x \in \bar{U}:(x, \Phi(x)) \cap\left(x, H^{J}(x, \mu(x))\right) \neq \varnothing\right\}=\left\{x \in \bar{U}:(x, \Phi(x)) \cap\left(x, H^{J}(x, 1)\right) \neq \varnothing\right\} \\
& =\left(J^{\star}\right)^{-1}(B)
\end{aligned}
$$

so from (17) we have $d\left(\left(G^{\star}\right)^{-1}(B)\right)=d\left(\left(J^{\star}\right)^{-1}(B)\right) \neq d(\varnothing)$. Now combine with the above and we have $d\left(\left(F^{\star}\right)^{-1}(B)\right)=d\left(\left(J^{\star}\right)^{-1}(B)\right) \neq d(\varnothing)$.

Also note one could adjust the proof in Theorem 5 if we use $\cong$ in $A_{\partial U}(\bar{U}, E)$ in Remark 2 .

In our next theorem $E$ will be a completely regular topological space and $U$ will be an open subset of $E$.

Theorem 6. Let $B=\{(x, \Phi(x)): x \in \bar{U}\}$, $d$ is defined in (15) and assume (2) and (3) hold. Suppose F and $G$ are two maps in $A_{\partial U}(\bar{U}, E)$ (write $F^{\star}=I \times F$ and $\left.G^{\star}=I \times G\right)$ and $F \cong G$ in $A_{\partial U}(\bar{U}, E)$. Then $F^{\star}$ is $d-\Phi-e s s e n t i a l$ if and only if $G^{\star}$ is $d-\Phi-e s s e n t i a l$.

Proof. In the proof below we assume $\cong$ in $A_{\partial U}(\bar{U}, E)$ is as in Definition 3. Assume $G^{\star}$ is $d-\Phi-$ essential. Let $J \in A_{\partial U}(\bar{U}, E)$ (write $J^{\star}=I \times J$ ) and $\left.J\right|_{\partial U}=\left.F\right|_{\partial U}$. If we show (16) then $F^{\star}$ is $d-\Phi$-essential from Theorem 5. Now (3) implies $J \cong F$ in $A_{\partial U}(\bar{U}, E)$ and this together with $F \cong G$ in $A_{\partial U}(\bar{U}, E)$ and (2) guarantees that $G \cong J$ in $A_{\partial U}(\bar{U}, E)$. It remains to show $d\left(\left(F^{\star}\right)^{-1}(B)\right)=d\left(\left(G^{\star}\right)^{-1}(B)\right)$. Note since $G \cong F$ in $A_{\partial U}(\bar{U}, E)$ let $H: \bar{U} \times[0,1] \rightarrow K(E)$ be a u.s.c. compact map with $H(\cdot, \eta(\cdot)) \in$ $\mathbf{A}(\bar{U}, E)$ for any continuous function $\eta: \bar{U} \rightarrow[0,1]$ with $\eta(\partial U)=0, \Phi(x) \cap H_{t}(x)=\varnothing$ for any $x \in \partial U$ and $t \in(0,1)$ (here $\left.H_{t}(x)=H(x, t)\right), H_{0}=G$ and $H_{1}=F$. Let $H^{\star}: \bar{U} \times[0,1] \rightarrow K(\bar{U} \times E)$ be given by $H^{\star}(x, t)=(x, H(x, t))$ and let

$$
K=\left\{x \in \bar{U}:(x, \Phi(x)) \cap H^{\star}(x, t) \neq \varnothing \text { for some } t \in[0,1]\right\} .
$$

Now $K \neq \varnothing$ and there exists a Urysohn map $\mu: \bar{U} \rightarrow[0,1]$ with $\mu(\partial U)=0$ and $\mu(K)=1$. Let $R(x)=H(x, \mu(x))$ and write $R^{\star}=I \times R$. Now $R \in A_{\partial U}(\bar{U}, E)$ with $\left.R\right|_{\partial U}=\left.G\right|_{\partial U}$ so since $G^{\star}$ is $d$ - $\Phi$-essential then $d\left(\left(G^{\star}\right)^{-1}(B)\right)=d\left(\left(R^{\star}\right)^{-1}(B)\right) \neq d(\varnothing)$. Now since $\mu(K)=1$ we have

$$
\begin{aligned}
& \begin{aligned}
\left(R^{\star}\right)^{-1}(B) & =\{x \in \bar{U}:(x, \Phi(x)) \cap(x, H(x, \mu(x))) \neq \varnothing\}=\{x \in \bar{U}:(x, \Phi(x)) \cap(x, H(x, 1)) \neq \varnothing\} \\
& =\left(F^{\star}\right)^{-1}(B),
\end{aligned} \\
& \text { sod }\left(\left(F^{\star}\right)^{-1}(B)\right)=d\left(\left(G^{\star}\right)^{-1}(B)\right) .
\end{aligned}
$$

Also note one could adjust the proof in Theorem 6 if we use $\cong$ in $A_{\partial U}(\bar{U}, E)$ in Remark 2 .

Remark 8. It is very easy to extend the above ideas to the $(L, T) d-\Phi-e s s e n t i a l$ maps in [3].

Funding: This research received no external funding.

Conflicts of Interest: The author declares no conflict of interest. 


\section{References}

1. Granas, A. Sur la méthode de continuité de Poincaré. C. R. Acad. Sci. 1976, 282, 983-985.

2. O'Regan, D. Generalized coincidence theory for set-valued maps. J. Nonlinear Sci. Appl. 2017, 10, 855-864. [CrossRef]

3. O'Regan, D. Topological transversality principles and general coincidence theory. An. Stiint. Univ. Ovidius Constanta Ser. Math. 2017, 25, 159-170. [CrossRef]

4. $\quad$ Engelking, R. General Topology; Heldermann Verlag: Berlin, Germany, 1989.

5. Aliprantis, C.D.; Border, K.C. Studies in Economic Theory. In Infinite-Dimensional Analysis; Springer: Berlin, Germany, 1994; Volume 4.

6. Eilenberg, S.; Montgomery, D. Fixed point theory for multivalued transformations. Am. J. Math. 1946, 68, 214-222. [CrossRef]

7. Fitzpatrick, P.M.; Petryshyn, W.V. Fixed point theory for multivalued noncompact acyclic mappings. Pac. J. Math. 1974, 54, 12-23. [CrossRef]

(c) 2020 by the author. Licensee MDPI, Basel, Switzerland. This article is an open access article distributed under the terms and conditions of the Creative Commons Attribution (CC BY) license (http:/ / creativecommons.org/licenses/by/4.0/). 